\title{
Qualidade de vida de crianças e adolescentes com câncer: Estudos que aplicaram o PedsQL 3.0 Cancer Module
}

\author{
Quality of life in children and adolescents with cancer: Studies that applied the PedsQL 3.0 Cancer \\ Module
}

Calidad de vida de niños y adolescentes con cáncer: Estudios que aplicaron el Módulo de Cáncer

PedsQL 3.0

\author{
Roberta Dantas Breia de Noronha \\ ORCID: https://orcid.org/0000-0003-4495-6680 \\ Instituto Nacional de Câncer, Brasil \\ E-mail: robertadantasbn@yahoo.com.br \\ Maria Aparecida de Luca Nascimento \\ ORCID: https://orcid.org/0000-0003-0558-460X \\ Universidade Federal do Estado do Rio de Janeiro, Brasil \\ E-mail: gemeas@centroin.com.br \\ Sima Esther Ferman \\ ORCID: https://orcid.org/0000-0002-7076-6779 \\ Instituto Nacional de Câncer, Brasil \\ E-mail: sferman@uol.com.br \\ Sandra Alves do Carmo \\ ORCID: https://orcid.org/0000-0001-8413-0053 \\ Universidade Federal do Estado do Rio de Janeiro, Brasil \\ E-mail: drinhaalves@yahoo.com.br
}

\begin{abstract}
Resumo
Objetivo: apontar a qualidade de vida de crianças e adolescentes com câncer de estudos que aplicaram o PedsQL 3.0 Cancer Module e descrever o escore encontrado nesses estudos. Metodologia: Revisão sistemática da literatura nas bases de dados Medline, LILACS, Scielo, Scopus, PsycINFO, Science Direct e Web of Science de estudos publicados entre 2015 e 2020. Resultados: Selecionou-se 18 artigos, onde observa-se que a Leucemia foi o tipo de câncer mais frequente em $78 \%$ dos estudos. A quimioterapia foi o tratamento recebido em $55 \%$ das pesquisas e em relação ao escore médio dos participantes, consta-se que os escores variaram entre 59,1 e 74,53. Conclusão: a média de escore variou entre 59,1 e 74,53. O tipo de câncer parece não ter influência significativa nos escores. A fadiga é o sintoma mais associado aos baixos escores de qualidade de vida. E pacientes tratados com quimioterapia tendem a apresentar menores escores de qualidade de vida.
\end{abstract}

Palavras-chave: Inquéritos e questionários; Neoplasias; Qualidade de vida; Saúde da criança.

\begin{abstract}
Objective: to point the quality of life of children and adolescents with cancer from studies that applied the PedsQL 3.0 Cancer Module and describe the score found in these studies. Methodology: Systematic literature review in the databases Medline, LILACS, Scielo, Scopus, PsycINFO, Science Direct and Web of Science of studies published between 2015 and 2020. Results: 18 articles were selected, where it was observed that Leukemia was the most frequent type of cancer in $78 \%$ of the studies. Chemotherapy was the treatment received in 55\% of the research and in relation to the average score of the participants, it is found that the scores ranged from 59.1 to 74.53 . Conclusion: The mean score ranged from 59.1 to 74.53. The type of cancer seems to have no significant influence on the scores. Fatigue is the symptom most associated with low quality of life scores. And patients treated with chemotherapy tend to have lower quality of life scores.
\end{abstract}

Keywords: Surveys and questionnaires; Neoplasms; Quality of life; Child health.

\section{Resumen}

Objetivo: apuntar la calidad de vida de los niños y adolescentes con cáncer a partir de los estudios que aplicaron el Módulo de Cáncer PedsQL 3.0 y describir la puntuación encontrada en estos estudios. Metodología: Revisión sistemática de la literatura en las bases de datos Medline, LILACS, Scielo, Scopus, PsycINFO, Science Direct y Web of Science de los estudios publicados entre 2015 y 2020. Resultados: Se seleccionaron 18 artículos, donde se observó que la leucemia era el tipo de cáncer más frecuente en el $78 \%$ de los estudios. La quimioterapia fue el tratamiento 
recibido en el 55\% de las investigaciones y en relación con la puntuación media de los participantes, se encuentra que las puntuaciones oscilaron entre 59,1 y 74,53. Conclusión: la puntuación media osciló entre 59,1 y 74,53. El tipo de cáncer no parece tener una influencia significativa en las puntuaciones. La fatiga es el síntoma que más se asocia a las puntuaciones bajas de calidad de vida. Los pacientes tratados con quimioterapia tienden a tener puntuaciones de calidad de vida más bajas.

Palabras clave: Encuestas y cuestionarios; Neoplasias; Calidad de vida; Salud del niño.

\section{Introdução}

O Pediatric Quality of Life Inventory ${ }^{T M} 3.0$ - Cancer Module (PedsQL 3.0) é um questionário desenvolvido para mensurar a qualidade de vida de crianças e adolescentes com câncer, que inclui uma versão de autorrelato e de heterorrelato dos pais. A avaliação das crianças inclui as seguintes faixas etárias: 05 a 07; 08 a 12 e 13 a 18 anos. O questionário dos pais inclui as faixas etárias de 02 a 04; 05 a 07; 08 a 12 e 13 a 18 anos (Varni, 2017).

De acordo com Varni (2017), o questionário é composto por 27 itens, categorizados em oito subescalas: "dor” (dois itens); "náusea" (cinco itens); "ansiedade nos procedimentos" (três itens); "ansiedade nos tratamentos" (três itens); "preocupação" (três itens); "problemas cognitivos" (cinco itens); "percepção da aparência física" (três itens); e "comunicação" (três itens).

As questões indagam ao indivíduo o quanto cada item representou um problema no último mês, com uma escala de respostas, tipo Likert de cinco opções ( 0 - nunca é um problema; 1 - quase nunca é um problema; 2 - algumas vezes é um problema; 3 - frequentemente é problema; 4 - quase sempre é um problema). Perguntas negativas são pontuadas inversamente em uma escala de 0-100; assim, quanto maior o escore, menor serão as dificuldades enfrentadas em relação a doença e/ou tratamento, ou seja, melhor a qualidade de vida (Varni, 2017).

$O$ PedsQL 3.0 possibilita detectar particularidades da qualidade de vida relacionada à doença e ao tratamento das crianças e adolescentes acometidos com câncer. Sendo assim, este estudo objetiva apontar a qualidade de vida de crianças e adolescentes com câncer de estudos que aplicaram o PedsQL 3.0 Cancer Module e descrever o escore encontrado nesses estudos (Varni, 2017).

\section{Metodologia}

Foi realizada uma revisão sistemática da literatura em acordo com as diretrizes propostas pelo Preferred Reporting Items for Systematic Reviews and Meta-Analyses -PRISMA (Moher, 2009) a partir da seguinte pergunta: Qual a qualidade de vida de crianças e adolescentes com câncer avaliadas através do Módulo de Câncer PedsQL 3.0 apontada nas produções científicas?

Com o intuito de localizar as principais produções científicas nacionais e internacionais, foram realizadas buscas por artigos científicos em agosto de 2020 em sete bases de dados: Medline, Literatura Latino-Americana e do Caribe em Ciências da Saúde (LILACS), Scientific Eletronic Library Online (Scielo), Scopus, PsycINFO, Science Direct e Web of Science. A chave de descritores utilizada nas bases de dados foi: (surveys and questionnaires) and ("quality of life") and (cancer) and ("child health").

Embora a mesma chave de descritores e tipo de filtros (i.e. tipo de documento e ano) tenham sido utilizados em todas as bases eletrônicas, as estratégias de buscas foram customizadas em acordo com o funcionamento de cada uma das bases de dados. Sobretudo no que diz respeito à nomenclatura do filtro "tipo de documento" o qual varia em cada uma das bases de dados: Medline, Lilacs, Scielo, Scopus e Web of Science (Article), Science Direct (Research articles), PsycINFO (Journals). Em relação ao filtro "ano", em todas as bases de dados foram selecionados apenas os estudos publicados entre 2015 e 2020. 
Após aplicação desses filtros, todos os artigos localizados foram importados ao website "Rayyan" onde foram conduzidas as etapas de seleção e elegibilidade dos artigos conforme diretrizes do PRISMA. O Rayyan é uma ferramenta online e gratuita, similar ao Covidence (2020) e desenvolvida para auxiliar os pesquisadores a realizarem revisões sistemáticas com ou sem metanálise de forma ágil e com menor risco de viés de seleção, pois permite que os resultados das buscas sejam armazenados em uma única base de dados online onde os juízes podem realizar a avaliação dos artigos às cegas. Ao término da seleção de artigos o website permite a remoção do blind mode, exibindo quantos artigos foram incluídos por cada juiz e se existem ou não divergências de opinião sobre a decisão de incluir ou excluir determinado estudo. Além disso, o Rayyan é capaz de identificar e eliminar os artigos repetidos entre as bases de dados (Ouzzani, et al. 2016).

Os critérios de inclusão utilizados para seleção dos artigos foram: I) artigos empíricos publicados entre 2015 e 2020; II); com amostra composta por crianças e adolescentes com diagnóstico de câncer; III) que avaliaram a qualidade de vida dos participantes utilizando o instrumento Pediatric Quality of Life Inventory (PedsQL) modulo câncer 3.0.

Os critérios de exclusão foram: I) artigos repetidos; II) amostras compostas exclusivamente por adultos ou idosos; III) estudos com amostras mistas (i.e. crianças, adolescentes e adultos) que não apresentarem resultados separados por faixa etária; IV) artigos que não reportaram a versão/modulo utilizado no PedsQL; V) estudos que utilizaram amostras compostas exclusivamente por sobreviventes de câncer; VI) produções acadêmicas que não passaram pelo processo de revisão por pares (e.g. teses, dissertações, trabalhos de conclusão, livros e anais de eventos científicos).

Não foram realizadas restrições relacionadas ao idioma das publicações visando abranger o maior número de artigos possíveis sobre a temática em estudo. Para aplicação dos critérios de inclusão e exclusão, foi realizada inicialmente a leitura dos títulos e resumos de cada um dos artigos localizados. Nessa etapa, excluíram-se os artigos que não apresentavam nenhum dos descritores utilizados nas buscas em seus títulos e resumos. Posteriormente, durante a etapa de leitura na integra, foram eliminados os estudos que não respondiam ao objetivo desta revisão ou que não se adequaram aos critérios pré-estabelecidos.

\section{Resultados}

Inicialmente foram localizados 607 artigos distribuídos entre as bases de dados: Medline $(n=391)$, Lilacs $(n=12)$, Scopus $(n=29)$, Web of Science $(n=10)$, Science Direct $(n=135)$, PsycINFO $(n=28)$ e Scielo $(n=2)$. A Figura 1 demonstra detalhadamente as etapas utilizadas para exclusão dos artigos conforme as diretrizes propostas pelo PRISMA. 
Figura 1. Fluxog5ama do processo de seleção dos artigos.

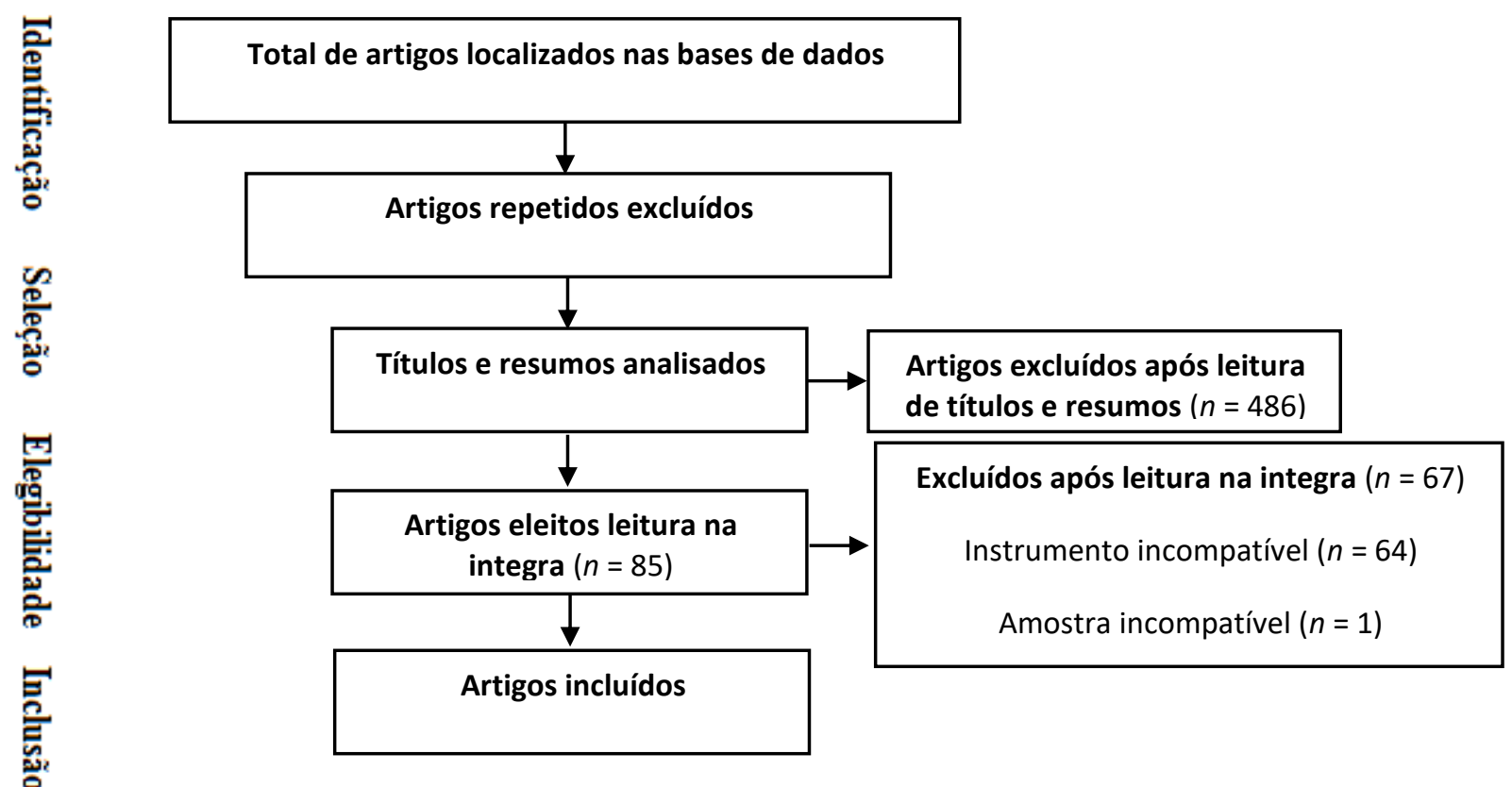

Fonte: Autores.

A análise dos resultados foi realizada por meio da extração e síntese das principais informações relacionadas aos 18 artigos incluídos. Essa síntese é apresentada em forma de quadros de resultados. O Quadro 1 apresenta a caracterização dos artigos incluídos: Número do artigo, Bases de dados, Autores, Ano de publicação e periódico onde o artigo foi publicado, País de origem dos participantes, Título, Nível de evidência, Objetivo, Tipo de Estudo e principais resultados.

Cada um dos 18 artigos incluídos recebeu um número em ordem crescente $(1,2,3,4 \ldots)$. Essa numeração é utilizada para fazer menção aos estudos incluídos em outro quadro e ao longo deste artigo. Em relação ao nível de evidência científica, todos os artigos incluídos foram classificados dentre sete possíveis níveis de acordo com o guia para melhores práticas em enfermagem: Nível I - Revisões sistemáticas ou metanálises de ensaios clínicos randomizados (ECR); Nível II - ECR controlado bem delineado; Nível III - Ensaios clínicos bem delineados, mas sem randomização; Nível IV - Estudos de coorte ou caso-controle; Nível V - Revisão sistemática de estudos descritivos ou qualitativos; Nível VI - Estudo descritivo ou qualitativo; Nível VII - Opinião de autoridades ou relatório de comitês de especialistas ${ }^{4}$. Destaca-se que não foram realizadas classificações considerando os níveis de evidência I, V e VII devido à exclusão de estudos não empíricos, conforme critérios de inclusão e exclusão desta revisão sistemática (Melnyk, 2011).

Tendo como base os dados expostos no Quadro 1, constata-se que 78\% $(\mathrm{N}=14)$ dos artigos incluídos nesta revisão foram recuperados da base de dados MEDLINE. O restante dos artigos incluídos estava indexado na Scopus ( $\mathrm{N}=2$ ) e LILACS $(\mathrm{N}=2)$, equivalente a $11 \%$ em cada base. Não foram localizados artigos que respondessem ao objetivo desta revisão na Web of Science, Science Direct, PsycINFO e Scielo. No que diz respeito ao período das publicações selecionadas nesta revisão (20152020), observa-se que 2017 foi o ano com o maior número de publicações, cerca de $39 \%$ dos artigos ( $\mathrm{N}=7$ ), seguido de 2015 e 2019 com 22,3\% ( $\mathrm{N}=4)$ em cada ano. Apenas 11\% ( $\mathrm{N}=2)$ dos estudos foram publicados em 2018 e apenas um em 2016 (5,6\%). Considerando que foram localizados menos artigos em 2018 e 2019 em comparação a 2017 e que não foram identificados artigos de 2020, infere-se a possibilidade de uma redução no número de publicações utilizando o módulo câncer do PedsQL. 
Em relação aos periódicos em que os artigos estão publicados, todos os estudos são provenientes de 15 periódicos. Dentre esses, apenas três periódicos (Cancer Nursing; Journal of Pediatric Oncology e European Journal of Oncology Nursing) publicaram dois estudos cada. No que tange aos países que conduziram os estudos, a maior parte das pesquisas foram conduzidas nos EUA e no Brasil, cada um representando 22,3\% ( $\mathrm{N}=4)$ do total, na sequência aparecem China e Canada 11\% $(\mathrm{N}=2)$ cada. As seis pesquisas restantes foram realizadas em países distintos (i.e. México, Australia, Holanda, Japão, Hong Kong e Colombia). Embora o local de realização das pesquisas tenha variado, cerca de $78 \%(\mathrm{~N}=14)$ dos artigos foram redigidos em inglês, somente $11 \%(\mathrm{~N}=2)$ em português e outros $11 \%(\mathrm{~N}=2)$ em espanhol.

No que diz respeito ao nível de evidência dos artigos incluídos, observa-se que a maior parte dos estudos $72 \%(\mathrm{~N}=$ 13) eram de nível VI, seguidos dos estudos de nível IV que representam 16,7\% ( N = 3) e 11\% ( $\mathrm{N}=2$ ) de nível II. O Quadro 2 demonstra as principais características dos participantes dos 18 estudos incluídos: Número de participantes, Sexo, Idade, Tipo de câncer, Tratamento e Escore médio do PedsQL 3.0 Cancer Module. 
Quadro 1 - Caracterização dos estudos incluídos.

\begin{tabular}{|c|c|c|c|c|c|c|c|}
\hline $\mathbf{N}^{\circ}$ & Bases de Dados & $\begin{array}{c}\text { Autores/Ano/ } \\
\text { Revista/País }\end{array}$ & Título & Evidência & Objetivo & $\begin{array}{l}\text { Tipo de } \\
\text { estudo }\end{array}$ & Resultados \\
\hline 1 & MEDLINE & $\begin{array}{c}\text { Stinson et } \\
\text { al./(2015)/ Pain/ } \\
\text { Canada }\end{array}$ & $\begin{array}{l}\text { Construct validity and } \\
\text { reliability of a real-time } \\
\text { multidimensional } \\
\text { smartphone app to assess } \\
\text { pain in children and } \\
\text { adolescents with cancer }\end{array}$ & Nível VI & $\begin{array}{c}\text { Avaliar a validade de } \\
\text { construto, confiabilidade e } \\
\text { viabilidade do aplicativo de } \\
\text { smartphone multidimensional } \\
\text { (Pain Squad) para avaliação da } \\
\text { dor em crianças e adolescentes } \\
\text { com câncer }\end{array}$ & $\begin{array}{c}\text { Descritivo } \\
\text { Transversal }\end{array}$ & $\begin{array}{c}\text { Os resultados fornecem } \\
\text { evidências da validade de } \\
\text { construto, confiabilidade e } \\
\text { viabilidade do aplicativo } \\
\text { Pain Squad para avaliação de } \\
\text { crianças e adolescentes com } \\
\text { câncer } \\
\end{array}$ \\
\hline 3 & SCOPUS & $\begin{array}{l}\text { Rodgers et al./ } \\
\text { (2015)/ Journal } \\
\text { of Pediatric } \\
\text { Oncology } \\
\text { Nursing/ EUA }\end{array}$ & $\begin{array}{l}\text { Health-Related Quality of } \\
\text { Life among Children and } \\
\text { Adolescents during } \\
\text { Hematopoietic Stem Cell } \\
\text { Transplant Recovery }\end{array}$ & Nível VI & $\begin{array}{l}\text { Descrever as mudanças de } \\
\text { QVRS entre crianças e } \\
\text { adolescentes durante os } \\
\text { primeiros } 6 \text { meses de } \\
\text { recuperação de transplante de } \\
\text { células tronco e avaliar as } \\
\text { associações entre fatores } \\
\text { demográficos, diagnóstico, } \\
\text { informações de transplante, e } \\
\text { sintomas com QVRS }\end{array}$ & $\begin{array}{c}\text { Descritivo } \\
\text { Transversal }\end{array}$ & $\begin{array}{c}\text { Nenhuma diferença } \\
\text { significativa nos escores de } \\
\text { QVRS foi observada entre } \\
\text { fatores demográficos, } \\
\text { diagnósticos ou de } \\
\text { transplante. Sentir-se } \\
\text { cansado, triste, preocupado } \\
\text { ou ter insônia no primeiro } \\
\text { mês pós-transplante foi } \\
\text { negativamente } \\
\text { correlacionado com QVRS }\end{array}$ \\
\hline 4 & MEDLINE & $\begin{array}{c}\text { Place et al./ } \\
\text { (2015)/ Lancet } \\
\text { Oncology/ EUA }\end{array}$ & $\begin{array}{c}\text { Intravenous pegylated } \\
\text { asparaginase versus } \\
\text { intramuscular native } \\
\text { Escherichia coli L- } \\
\text { asparaginase in newly } \\
\text { diagnosed childhood acute } \\
\text { lymphoblastic leukaemia } \\
\text { (DFCI 05-001): a } \\
\text { randomised, open-label } \\
\text { phase } 3 \text { trial }\end{array}$ & Nível II & $\begin{array}{l}\text { Comparar a toxicidade relativa } \\
\text { e eficácia de PEG-asparaginase } \\
\text { intravenosa e Ecoli l- } \\
\text { asparaginase intramuscular } \\
\text { nativa em crianças com } \\
\text { diagnóstico recente Leucemia } \\
\text { linfoide aguda (LLA) }\end{array}$ & $\begin{array}{c}\text { Estudo } \\
\text { Randomizado } \\
\text { Controlado }\end{array}$ & $\begin{array}{c}\text { A PEG-asparaginase } \\
\text { intravenosa não foi mais } \\
\text { tóxica do que, foi } \\
\text { similarmente eficaz e foi } \\
\text { associada com diminuição da } \\
\text { ansiedade em comparação } \\
\text { com E coli l-asparaginase } \\
\text { intramuscular nativa }\end{array}$ \\
\hline
\end{tabular}




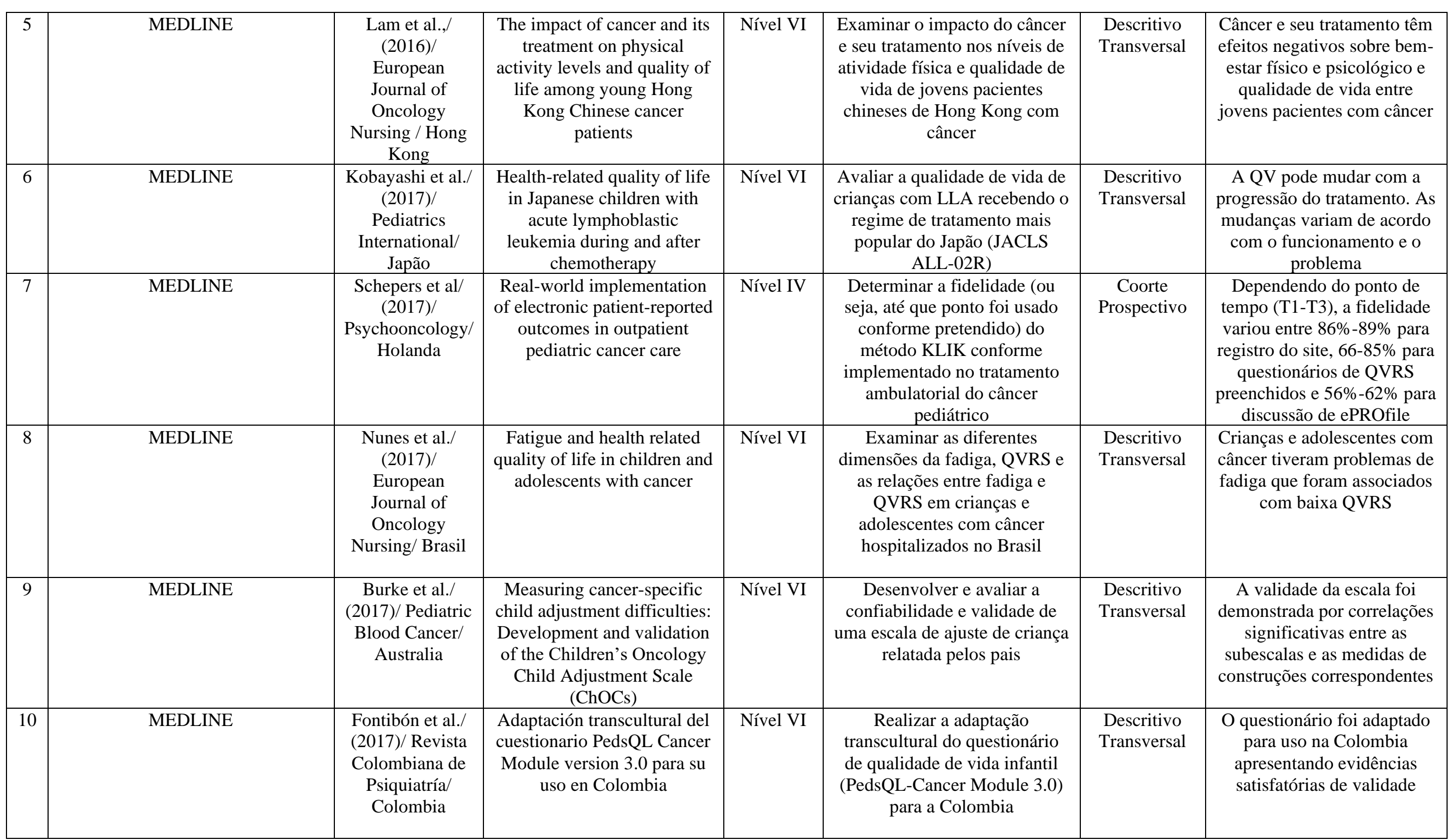




\begin{tabular}{|c|c|c|c|c|c|c|c|}
\hline 11 & LILACS & $\begin{array}{c}\text { Oliveira et al./ } \\
\text { (2017)/ Revista } \\
\text { da Sociedade } \\
\text { Brasileira de } \\
\text { Clínica Médica/ } \\
\text { Brasil } \\
\end{array}$ & $\begin{array}{l}\text { Cuidados paliativos: } \\
\text { prevalência de fadiga em } \\
\text { pacientes pediátricos }\end{array}$ & Nível VI & $\begin{array}{l}\text { Avaliar fadiga e qualidade de } \\
\text { vida em pacientes pediátricos } \\
\text { oncológicos internados em } \\
\text { enfermaria, sob a óptica dos } \\
\text { cuidados paliativos }\end{array}$ & $\begin{array}{l}\text { Descritivo } \\
\text { Transversal }\end{array}$ & $\begin{array}{l}\text { Verificou-se baixa } \\
\text { prevalência de fadiga na } \\
\text { amostra estudada e boa } \\
\text { qualidade de vida } \\
\text { relacionada à saúde. }\end{array}$ \\
\hline 12 & SCOPUS & $\begin{array}{c}\text { Carneiro et al/ } \\
\text { (2017)/ Journal } \\
\text { of Public Health/ } \\
\text { Brasil }\end{array}$ & $\begin{array}{l}\text { Factors associated with } \\
\text { health-related quality of life } \\
\text { among children with cancer } \\
\text { from the standpoint of } \\
\text { patients and caregivers }\end{array}$ & Nível VI & $\begin{array}{l}\text { Avaliar os fatores associados } \\
\text { com QVRS em crianças com } \\
\text { câncer do ponto de vista de } \\
\text { pacientes e cuidadores }\end{array}$ & $\begin{array}{c}\text { Descritivo } \\
\text { Transversal }\end{array}$ & $\begin{array}{l}\text { Os relatos de sintomas do } \\
\text { paciente e do cuidador } \\
\text { produziram resultados } \\
\text { distintos, embora } \\
\text { dificuldades cognitivas e } \\
\text { sorrir com vergonha foram } \\
\text { significativamente } \\
\text { associados com qualidade de } \\
\text { vida prejudicada em ambos } \\
\text { relatos }\end{array}$ \\
\hline 13 & MEDLINE & $\begin{array}{l}\text { Crane et al./ } \\
\text { (2018)/ Journal } \\
\text { of Pediatric } \\
\text { Oncology } \\
\text { Nursing / EUA }\end{array}$ & $\begin{array}{c}\text { Understanding Treatment } \\
\text { Burden and Quality of Life } \\
\text { Impact of Participating in an } \\
\text { Early-Phase Pediatric } \\
\text { Oncology Clinical Trial: A } \\
\text { Pilot Study }\end{array}$ & Nível IV & $\begin{array}{l}\text { Avaliar a viabilidade e os } \\
\text { resultados preliminares de ter } \\
\text { medidas completadas por } \\
\text { crianças com câncer e seus } \\
\text { pais de carga de tratamento e } \\
\text { qualidade de vida (QV) } \\
\text { simultaneamente com } \\
\text { participação no EPT }\end{array}$ & $\begin{array}{c}\text { Coorte } \\
\text { Prospectivo }\end{array}$ & $\begin{array}{l}\text { Metas de viabilidade de } \\
\text { inscrição, retençãa e } \\
\text { conclusão de medição foram } \\
\text { parcialmente atendidos. A } \\
\text { carga de tratamento } \\
\text { preliminar e os resultados de } \\
\text { QV foram fornecidos }\end{array}$ \\
\hline 14 & LILACS & $\begin{array}{c}\text { Costa et al./ } \\
\text { (2018)/ Revista } \\
\text { brasileira de } \\
\text { cancerologia/ } \\
\text { Brasil }\end{array}$ & $\begin{array}{c}\text { Avaliação da Força de } \\
\text { Preensão Palmar e } \\
\text { Qualidade de Vida de } \\
\text { Crianças com Câncer } \\
\text { Submetidas à Quimioterapia } \\
\text { com Vincristina }\end{array}$ & Nível VI & $\begin{array}{l}\text { Avaliar a força de preensão } \\
\text { palmar e a qualidade de vida } \\
\text { de crianças e adolescentes com } \\
\text { câncer submetidos à } \\
\text { quimioterapia com vincristina }\end{array}$ & $\begin{array}{l}\text { Descritivo } \\
\text { Transversal }\end{array}$ & $\begin{array}{c}\text { A quimioterapia com } \\
\text { vincristina reduz a força } \\
\text { muscular periférica em } \\
\text { pacientes com câncer, nos } 30 \\
\text { primeiros dias. Em relação à } \\
\text { qualidade de vida, não foi } \\
\text { apresentada diferença } \\
\text { significativa }\end{array}$ \\
\hline 15 & MEDLINE & $\begin{array}{c}\text { Xia et al./ } \\
\text { (2019)/ } \\
\text { Hematologic } \\
\text { Cancer and } \\
\text { Quality of Life/ } \\
\text { Cancer Nursing/ } \\
\end{array}$ & $\begin{array}{c}\text { The Impact of Hematologic } \\
\text { Cancer and Its Treatment on } \\
\text { Physical Activity Level and } \\
\text { Quality of Life Among } \\
\text { Children in Mainland China }\end{array}$ & Nível VI & $\begin{array}{c}\text { Examinar os efeitos do câncer } \\
\text { hematológico e seu tratamento } \\
\text { na atividade física e QV entre } \\
\text { as crianças chinesas }\end{array}$ & $\begin{array}{c}\text { Descritivo } \\
\text { Transversal }\end{array}$ & $\begin{array}{l}\text { O nível de atividade física e } \\
\text { a QV em crianças com } \\
\text { câncer hematológico foram } \\
\text { menores do que em crianças } \\
\text { saudáveis }\end{array}$ \\
\hline
\end{tabular}


Research, Society and Development, v. 10, n. 8, e52710817570, 2021

(CC BY 4.0) | ISSN 2525-3409 | DOI: http://dx.doi.org/10.33448/rsd-v10i8.17570

\begin{tabular}{|c|c|c|c|c|c|c|c|}
\hline & & China & & & & & \\
\hline 16 & MEDLINE & $\begin{array}{l}\text { Rodgers et al./ } \\
(2019) / \\
\text { Oncology } \\
\text { Nursing Forum/ } \\
\text { EUA } \\
\end{array}$ & $\begin{array}{l}\text { Childhood Cancer Symptom } \\
\text { Cluster: Leukemia and } \\
\text { Health-Related Quality of } \\
\text { Life }\end{array}$ & Nível IV & $\begin{array}{c}\text { Examinar a relação do Cluster } \\
\text { de sintomas de câncer infantil - } \\
\text { leucemia (CCSC-L) com } \\
\text { QVRS }\end{array}$ & Coorte & 16 \\
\hline 17 & MEDLINE & $\begin{array}{c}\text { Liu et } \mathrm{al}^{21} . / \\
\text { 2019/ Cancer } \\
\text { Nursing/ China }\end{array}$ & $\begin{array}{c}\text { Chinese Version of Pediatric } \\
\text { Patient-Reported Outcomes } \\
\text { Measurement Information } \\
\text { System Short Form } \\
\text { Measures }\end{array}$ & Nível VI & $\begin{array}{l}\text { Examinar a confiabilidade, } \\
\text { validade e estrutura fatorial das } \\
\text { medidas C-Ped-PROMIS em } \\
\text { crianças com câncer na China }\end{array}$ & $\begin{array}{c}\text { Descritivo } \\
\text { Transversal }\end{array}$ & $\begin{array}{c}\text { O C-Ped-PROMIS } \\
\text { demonstrou boa validade de } \\
\text { grupo e boa validade } \\
\text { concorrente medida por } \\
\text { correlações significativas } \\
\text { com a PedsQL }\end{array}$ \\
\hline 18 & MEDLINE & $\begin{array}{c}\text { Gutiérrez- } \\
\text { Vargas et al./ } \\
\text { (2019)/ Boletín } \\
\text { Médico del } \\
\text { Hospital Infantil } \\
\text { de México }\end{array}$ & $\begin{array}{c}\text { Validación de un } \\
\text { instrumento para medir la } \\
\text { calidad de vida en niño }\end{array}$ & Nível VI & $\begin{array}{c}\text { Traduzir, adaptar } \\
\text { transculturalmente e validar } \\
\text { uma nova versão em espanhol } \\
\text { do instrumento Mucosite } \\
\text { Orofaríngea - Qualidade de } \\
\text { Vida Específica (OMQoL) em } \\
\text { pacientes pediátricos }\end{array}$ & $\begin{array}{c}\text { Descritivo } \\
\text { Transversal }\end{array}$ & $\begin{array}{l}\text { A nova versão do OMQoL } \\
\text { em espanhol demonstrou } \\
\text { propriedades psicométricas } \\
\text { adequadas e é um } \\
\text { instrumento confiável e } \\
\text { válido para medir a QV em } \\
\text { crianças com MO }\end{array}$ \\
\hline
\end{tabular}

Fonte: Dados da Pesquisa (2020). 
Research, Society and Development, v. 10, n. 8, e52710817570, 2021

(CC BY 4.0) | ISSN 2525-3409 | DOI: http://dx.doi.org/10.33448/rsd-v10i8.17570

Quadro 2 - Principais características dos participantes dos estudos incluídos.

\begin{tabular}{|c|c|c|c|c|c|c|}
\hline $\mathbf{N}^{\circ}$ & Participantes & Sexo & $\begin{array}{l}\text { Faixa } \\
\text { Etária }\end{array}$ & Tipo de Câncer & Tratamento Recebido & $\begin{array}{c}\text { Escore médio PedsQL - } \\
\text { CM 3.0 (DP) }\end{array}$ \\
\hline 1 & 106 & $54 \mathrm{M} ; 52 \mathrm{~F}$ & $8-18$ & $\begin{array}{c}\text { LLA; LMA; LNH; } \\
\text { Osteossarcoma; Tumor no SNC; } \\
\text { SDE; OTC }\end{array}$ & Quimioterapia; Radioterapia; Cirurgia & $69,78(16,14)$ \\
\hline 2 & 231 & NR & $2-18$ & $\mathrm{NR}$ & NR & NR \\
\hline 3 & 23 & $15 \mathrm{M} ; 8 \mathrm{~F}$ & $7-18$ & Leucemia; Linfoma; SMD & Transplante de Células Tronco & $74,43(18,44)$ \\
\hline 4 & 463 & $255 \mathrm{M} ; 208 \mathrm{~F}$ & $1-18$ & LLA & PEG-asparaginase; native E coli l-asparaginase & $\mathrm{NR}$ \\
\hline 5 & 76 & $44 \mathrm{M} ; 32 \mathrm{~F}$ & $9-18$ & $\begin{array}{l}\text { Leucemia; Linfoma; Tumor no } \\
\text { SNC ou nos ossos; OTC }\end{array}$ & Quimioterapia; Cirurgia & $63,27(7,3)$ \\
\hline 6 & 35 & $16 \mathrm{M} ; 19 \mathrm{~F}$ & $5-18$ & LLA & JACLS ALL-02R & $60,3(18,9)$ \\
\hline 7 & 205 & $118 \mathrm{M} ; 87 \mathrm{~F}$ & $8-18$ & $\begin{array}{l}\text { Leucemia; Linfoma; Tumores } \\
\text { sólidos; Tumor no SNC }\end{array}$ & $\mathrm{NR}$ & $\mathrm{NR}$ \\
\hline 8 & 38 & $25 \mathrm{M} ; 13 \mathrm{~F}$ & $8-18$ & $\begin{array}{l}\text { Leucemia; Linfoma; Tumor no } \\
\text { SNC; Sarcoma; OTC }\end{array}$ & $\begin{array}{l}\text { Quimioterapia; Cirurgia; Quimioterapia + Cirurgia; Quimioterapia + } \\
\text { Radioterapia; Quimioterapia + Radioterapia + Cirurgia }\end{array}$ & $59,1(16,7)$ \\
\hline 9 & 132 & $64 \mathrm{M} ; 68 \mathrm{~F}$ & $2-10$ & $\begin{array}{l}\text { Linfoma; Tumores sólidos; } \\
\text { Tumor no SNC }\end{array}$ & NR & NR \\
\hline 10 & 20 & $12 \mathrm{M} ; 8 \mathrm{~F}$ & $2-18$ & $\begin{array}{l}\text { LLA; Tumor no SNC; Tumores } \\
\text { sólidos; Sarcoma; OTC }\end{array}$ & $\begin{array}{c}\text { Quimioterapia; Cirurgia; Quimioterapia + Cirurgia; Quimioterapia + } \\
\text { Radioterapia; Quimioterapia + Radioterapia + Cirurgia; Radioterapia + } \\
\text { Cirurgia }\end{array}$ & NR \\
\hline 11 & 37 & $22 \mathrm{M} ; 15 \mathrm{~F}$ & $5-18$ & $\begin{array}{l}\text { Leucemia; Linfoma; Tumor no } \\
\text { SNC; OTC }\end{array}$ & Quimioterapia; Cirurgia; Radioterapia; Quimioterapia + Radioterapia & NR \\
\hline 12 & 71 & $36 \mathrm{M} ; 35 \mathrm{~F}$ & $5-18$ & $\begin{array}{l}\text { Leucemia; Linfoma; Tumor no } \\
\text { SNC; Tumores sólidos }\end{array}$ & $\begin{array}{c}\text { Quimioterapia; Cirurgia; Radioterapia + Cirurgia; Quimioterapia + } \\
\text { Radioterapia; Quimioterapia + Cirurgia }\end{array}$ & $74,53(16,70)$ \\
\hline 13 & 13 & $4 \mathrm{M} ; 9 \mathrm{~F}$ & $4-20$ & $\mathrm{NR}$ & NR & $74(\mathrm{NR})$ \\
\hline 14 & 7 & $1 \mathrm{M} ; 6 \mathrm{~F}$ & $5-15$ & LLA; OTC & Quimioterapia & $65,74(\mathrm{NR})$ \\
\hline 15 & 125 & $74 \mathrm{M} ; 51 \mathrm{~F}$ & $9-16$ & Leucemia & Quimioterapia; Quimioterapia + Transplante de medula & NR \\
\hline 16 & 327 & $170 \mathrm{M} ; 157 \mathrm{~F}$ & $3-18$ & LLA & Quimioterapia & $70,08(15,66)$ \\
\hline 17 & 272 & $164 \mathrm{M} ; 108 \mathrm{~F}$ & $8-17$ & $\begin{array}{l}\text { Leucemia; Linfoma; Tumor no } \\
\text { SNC; Tumores sólidos }\end{array}$ & NR & NR \\
\hline 18 & 193 & 92M; 101F & $8-16$ & LLA; LNH; Osteosarcoma & $\begin{array}{l}\text { Quimioterapia; Radioterapia; Quimioterapia + Radioterapia; Transplante } \\
\text { de medula }\end{array}$ & NR \\
\hline
\end{tabular}

Nota: CM - Cancer Module; DP - Desvio Padrão; F - Feminino; LLA - Leucemia linfoide aguda; LNH - Linfoma não Hodgkin; LMA - Leucemia mieloide aguda; M - Masculino; NR - Não Reportado; SDE - Sarcoma de Ewing; SMD - Síndrome mielodisplásica; SNC - Sistema Nervoso Central; OTC - Outros tipos de câncer.

Fonte: Autores. 
Constata-se que o número de participantes dos artigos variou entre 7 e 463 . A soma dos participantes de todos os estudos chega a um total de 2374. Destaca-se que este número diz respeito a crianças e adolescentes com diagnóstico de câncer, não foram contabilizados o número de mães, pais e/ou crianças saudáveis utilizadas para comparação. Em relação ao sexo dos participantes, embora alguns dos estudos apresentem distribuição desproporcional, a soma de todos os estudos não apresenta diferença significativa na distribuição dos participantes por sexo, sendo 49,1\% ( $\mathrm{N}=1166)$ do sexo masculino e $50,9 \%(\mathrm{~N}=1208)$ do feminino.

No que tange os tipos de câncer, observa-se que a Leucemia foi o tipo de câncer mais frequente, reportado por $78 \%$ $(\mathrm{N}=14)$ dos estudos. O Linfoma e os Tumores no SNC também apresentaram alta prevalência, ambos reportados por 50\% ( $\mathrm{N}$ = 9) dos artigos incluídos. Dois estudos não reportaram o tipo de câncer dos participantes. Em relação ao tipo de tratamento recebido, a quimioterapia foi utilizada em $55 \%(\mathrm{~N}=10)$ das pesquisas. Na sequência aparece a cirurgia, reportada por $27,5 \%$ $(\mathrm{N}=5)$ estudos. Destaca-se que a maioria dos estudos utilizou mais de uma forma de tratamento. Além disso, 27,5\% $(\mathrm{N}=5)$, dos artigos incluídos, não reportaram o tipo de tratamento utilizado.

Em relação ao escore médio dos participantes de cada um dos estudos no PedsQL 3.0, constata-se que os escores variaram entre 59,1 e 74,53. No entanto, apesar de todos os artigos terem utilizado o PedsQL 3.0, recomenda-se que este resultado seja interpretado considerando duas limitações: 1) Metade dos artigos incluídos $(\mathrm{N}=9)$ não reportou o escore médio total obtido pelos participantes no PedsQL 3.0. Alguns desses estudos apresentaram somente correlações com outros instrumentos e/ou os escores de cada domínio da escala, sem o total. 2) Observa-se elevado desvio padrão em seis dos artigos $(66,6 \%)$ que reportaram o escore médio dos participantes. Outros dois estudos não reportaram o desvio padrão de suas mostras. Somente um dos artigos incluídos apresentou baixo desvio padrão entre os escores do PedsQL 3.0. Por fim, destaca-se que a maior parte desses escores estão relacionadas as percepções das crianças e adolescentes, os pais responderam apenas nos casos em que seus filhos tinham 6 anos ou menos.

\section{Discussão}

Os principais resultados encontrados sugerem que ocorreram variações significativas, nos escores do PedsQL 3.0, entre os estudos incluídos nesta revisão. Sendo assim, com o intuito de fornecer possíveis explicações para tais variações e realizar uma discussão relevante e organizada, elaboramos algumas categorias onde os artigos incluídos foram agrupados por similaridade de conteúdo e comparados com outros estudos da área.

\section{Tipos de câncer, sintomas e variação nos escores do PedsQL 3.0}

Apenas três dos artigos incluídos (Estudos 3, 5 e 8) avaliaram a relação entre tipo de câncer e escores do PedsQL 3.0. Somente o estudo 8 sugeriu que os pacientes com sarcoma tendem a apresentar menores escores de qualidade de vida. No entanto, dos nove artigos que avaliaram a associação entre sintomas e qualidade de vida, oito (estudos 3, 4, 6, 8, 12, 13, 14 e 16) identificaram menores escores no PedsQL 3.0 entre os pacientes que apresentavam sintomas de fadiga, sono, náusea, problemas cognitivos e ansiedade. Destaca-se que todos os estudos sugerem que a fatiga está associada a menor qualidade de vida. Sendo apontado pelo estudo 8 que os pacientes com sarcoma tendem a apresentar menores escores no PedsQL 3.0 justamente por apresentarem maiores escores de fadiga.

Tais achados, segundo Buffart et al. (2017), são similares aos de uma metanálises recente, a qual avaliou 34 ensaios clínicos randomizados e não identificou correlações entre diagnóstico e qualidade de vida. No que diz respeito aos sintomas quatro estudos também sugerem que os sintomas de depressão, fadiga e ansiedade são a principal causa de baixos escores de qualidade de vida, sendo que nos estudos de Ho et al. (2019), Nap-van der Vlist et al. (2019) e Ritt-Olson et al. (2018) foi 
utilizado o PedsQL e Duncan et al. (2017) utilizou outros instrumentos de mensuração. Ho et al. (2019), Nap-van der Vlist et al. (2019) e Su et al. (2018) ressaltam, ainda que a fadiga é o sintoma mais associado aos baixos escores de qualidade de vida.

No que diz respeito ao sintoma de náusea, embora seja um sintoma frequente, alguns autores sugerem que a náusea seria o quinto sintoma mais prevalente e incapacitante, ficando atrás dos sintomas de fadiga, dor e insônia. De acordo com Kokkonen et al. (2019), a fadiga e a insônia são mais prevalentes e incapacitantes do que a náusea. Observa-se assim que a literatura está em acordo com os achados de nossa revisão.

Em relação aos problemas cognitivos, Van Lonkhuizen et al. (2019) sugerem que tais sintomas estariam mais associados aos pacientes com câncer cerebral. No entanto, em nossa revisão, a maior parte dos estudos incluídos não evidenciou tal correlação. Embora os problemas cognitivos sejam frequentes e causem sofrimento significativo aos pacientes, Kokkonen et al. (2019) e Wayne et al. (2018) relatam que a fadiga, dor e distúrbios do sono (e.g. insônia) são os sintomas mais associados aos baixos escores de qualidade de vida dos jovens com câncer.

Em linhas gerais, observa-se que a variação nos escores de qualidade de vida, seja do PedsQL 3.0 ou de outros instrumentos, está mais associada ao número e intensidade dos sintomas do que com o tipo de diagnóstico. Ademais, a fadiga parece ser o sintoma mais associado aos baixos escores de qualidade de vida entre crianças e adolescentes com câncer.

\section{A relação entre o tipo de tratamento e a qualidade de vida}

Apenas quatro dos artigos incluídos (Estudos 8, 11, 12,15) avaliaram a relação entre tipo de tratamento recebido e os escores do PedsQL 3.0. A maior parte (Estudos 8, 11, 12) sugerem que os pacientes tratados com quimioterapia apresentam menores escores de qualidade de vida. Sendo observado ainda que quanto maior a duração da quimioterapia, piores são os escores de qualidade de vida. Tal redução dos escores de qualidade de vida está associada ao aumento dos sintomas de fadiga, sono, náusea, problemas cognitivos e ansiedade. Destaca-se ainda que os autores do Estudo 15 evidenciaram que os pacientes que recebiam quimioterapia e transplante de medula óssea apresentavam mais sintomas adversos em comparação aos pacientes tratados somente com quimioterapia.

Observa-se assim que a maior parte dos estudos incluídos, que avaliaram a relação entre tipo de tratamento e qualidade de vida, estão em acordo com a literatura, a qual comumente reporta que pacientes em tratamento com quimioterapia apresentam menores escores de qualidade de vida em comparação a outras formas de tratamento (Coça et al., 2019; Nunes et al., 2019; Skaczkowski et al. 2018).

Nesse sentido, Coça et al. (2019) e Skaczkowski et al. (2018) sugerem que indivíduos que recebem somente tratamento cirúrgico ou radioterápico apresentam melhores escores de qualidade de vida em comparação aos tratados com quimioterapia, seja de forma individual ou mesmo combinada (e.g. cirurgia + quimioterapia) (Nunes et al., 2018).

Destaca-se ainda que esses resultados permanecem estáveis independentemente do instrumento de mensuração utilizado, seja o PedsQL 3.0, ou outros instrumentos. Por fim, os estudos de Nunes et al. (2018 e 2019) sugerem que o escore médio das crianças e dos adolescentes com câncer tendem a variar entre 58 e 74, estando em acordo com os achados da presente revisão.

\section{Considerações Finais}

Os principais resultados desse estudo sugerem que a média de escore, no PedsQL 3.0, das crianças e adolescentes com câncer variou entre 59,1 e 74,53. Cabe ressaltar que apesar de todos os estudos dessa revisão utilizarem o PedsQL 3.0 para avaliar a qualidade de vida das crianças e dos adolescentes, a metade não reportou os escores médios encontrados em seus achados sendo essa uma limitação do estudo. 
Research, Society and Development, v. 10, n. 8, e52710817570, 2021

(CC BY 4.0) | ISSN 2525-3409 | DOI: http://dx.doi.org/10.33448/rsd-v10i8.17570

Observou-se que o tipo de câncer não teve influência significativa nos escores. A fadiga é o sintoma mais associado aos baixos escores de qualidade de vida. Além disso, os pacientes tratados com quimioterapia tendem a apresentar menores escores de qualidade de vida.

Evidenciou-se uma carência de estudos nacionais que utilizassem o PedsQL 3.0 para avaliação da qualidade de vida das crianças e dos adolescentes em tratamento para o câncer. Sendo assim, recomenda-se a realização de pesquisas nacionais que utilizem esse instrumento e correlacionem os escores de qualidade de vida com outras variáveis, como por exemplo, expectativa de vida com o tratamento e covariáveis sociodemográficas. A partir da melhor compreensão da relação entre qualidade de vida e perfil sociodemográfico das crianças e adolescentes com câncer, será possível elaborar políticas públicas e estratégias de intervenção mais direcionadas a públicos específicos.

\section{Referências}

Buffart, L. M., Kalter, J., Sweegers, M. G., Courneya, K. S., Newton, R. U., Aaronson, N. K., \& Steindorf, K. (2017). Effects and moderators of exercise on quality of life and physical function in patients with cancer: an individual patient data meta-analysis of 34 RCTs. Cancer treatment reviews, $52,91-104$.

Burke, K., McCarthy, M., Lowe, C., Sanders, M. R., Lloyd, E., Bowden, M., \& Williams, L. (2017). Measuring cancer-specific child adjustment difficulties: Development and validation of the Children's Oncology Child Adjustment Scale (ChOCs). Pediatric Blood \& Cancer, 64(3), e26223.

Carneiro, T. V., Ribeiro, I. L. A., Alves, C. V., Bonan, P. R. F., Neto, E. D. A. L., \& Valença, A. M. G. (2017). Factors associated with health-related quality of life among children with cancer from the standpoint of patients and caregivers. Journal of Public Health, 25(4), $371-377$.

Coça, K. L., Bergmann, A., Carrara de Angelis, E., Ferman, S., \& Ribeiro, M. G. (2019). Health-related quality of life of Brazilian children and adolescents with benign and malignant solid tumours: A prospective cohort study during the first year after hospital admission. European journal of cancer care, 28(5), e13102.

Costa, T. B. D., Frantzeski, M. H., Nascimento, D. M. D., \& Gregianin, L. J. (2018). Avaliação da força de preensão palmar e qualidade de vida de crianças com câncer submetidas à quimioterapia com vincristina. Revista brasileira de cancerologia, 64(325), 319-325.

Covidence (2020). Systematic review software. Veritas Health Innovation: Melbourne. https://www.covidence.org

Crane, S., Backus, L., Stockman, B., Carpenter, J. S., Lin, L., \& Haase, J. (2018). Understanding treatment burden and quality of life impact of participating in an early-phase pediatric oncology clinical trial: a pilot study. Journal of Pediatric Oncology Nursing, 35(1), 25-35.

Duncan, M., Moschopoulou, E., Herrington, E., Deane, J., Roylance, R., Jones, L., \& Taylor, S. C. (2017). Review of systematic reviews of nonpharmacological interventions to improve quality of life in cancer survivors. BMJ open, 7(11), 1-21.

Fontibón, L. F., Ardila, S. L., \& Sánchez, R. (2017). Adaptación transcultural del cuestionario PedsQL Cancer Module version 3.0 para su uso en Colombia. Revista Colombiana de Psiquiatría, 46(3), 161-167.

Gutiérrez-Vargas, R., Velasco-Rojano, E., Villasís-Keever, M. Á., Portilla-Robertson, J., Gutiérrez-Rodelo, A., Flores-Navarro, S., \& Zapata-Tarrés, M. (2019). Validación de un instrumento para medir la calidad de vida en niños con mucositis orofaríngea en tratamiento por cáncer. Boletín médico del Hospital Infantil de México. 76(1), 35-43.

Ho, K. Y., Li, W. H., Lam, K. W. K., Wei, X., Chiu, S. Y., Chan, C. F. G., \& Chung, O. K. J. (2019). Relationships among fatigue, physical activity, depressive symptoms, and quality of life in Chinese children and adolescents surviving cancer. European Journal of Oncology Nursing, $38,21-27$.

Johnston, D., Gerbing, R., Alonzo, T., Aplenc, R., Nagarajan, R., Schulte, F., \& Sung, L. (2015). Patient-reported outcome coordinator did not improve quality of life assessment response rates: a report from the Children's Oncology Group. PloS one, 10(4), e0125290.

Kobayashi, K., Nakagami-Yamaguchi, E., Hayakawa, A., Adachi, S., Hara, J., Tokimasa, S., \& Kuriyama, K. (2017). Health-related quality of life in Japanese children with acute lymphoblastic leukemia during and after chemotherapy. Pediatrics International, 59(2), 145-153.

Kokkonen, K., Tasmuth, T., Lehto, J. T., Kautiainen, H., Elme, A., Jääskeläinen, A. S., \& Saarto, T. (2019). Cancer patients' symptom burden and healthrelated quality of life (HRQoL) at tertiary cancer center from 2006 to 2013: a cross-sectional study. Anticancer research, 39(1), 271-77.

Lam, K. K., Li, W. H., Chiu, S. Y., \& Chan, G. C. (2016). The impact of cancer and its treatment on physical activity levels and quality of life among young Hong Kong Chinese cancer patients. European Journal of Oncology Nursing, 21, 83-89.

Liu, Y., Yuan, C., Wang, J., Shen, N., Shen, M., \& Hinds, P. S. (2019). Chinese version of pediatric patient-reported outcomes measurement information system short form measures: Reliability, validity, and factorial structure assessment in children with cancer in China. Cancer nursing, $42(6), 430-438$.

Melnyk, B. M., \& Fineout-Overholt, E. (2011). Evidence-based practice in nursing \& healthcare: A guide to best practice. Filadelfia: Lippincott Williams \& Wilkins.

Moher, D., Liberati, A., Tetzlaff, J., \& Altman, D. G. (2009). Preferred reporting itens for systematic reviews and meta-analyses: the PRISMA statement. Plos med., 6(7), e1000097. doi:10.1371/journal.pmed.1000097.g001. 
Research, Society and Development, v. 10, n. 8, e52710817570, 2021

(CC BY 4.0) | ISSN 2525-3409 | DOI: http://dx.doi.org/10.33448/rsd-v10i8.17570

Nap-van der Vlist, M. M., Dalmeijer, G. W., Grootenhuis, M. A., van der Ent, C. K., van den Heuvel-Eibrink, M. M., Wulffraat, N. M., \& Nijhof, S. L. (2019). Fatigue in childhood chronic disease. Archives of disease in childhood, 104(11), 1090-1095.

Nunes, M. D. R., Jacob, E., Bomfim, E. O., Lopes-Junior, L. C., de Lima, R. A. G., Floria-Santos, M., \& Nascimento, L. C. (2017). Fatigue and health related quality of life in children and adolescents with cancer. European Journal of Oncology Nursing, 29, 39-46.

Nunes, M. D. R., Nascimento, L. C., Fernandes, A. M., Batalha, L., De Campos, C., Gonçalves, A., \& Jacob, E. (2019). Pain, sleep patterns and health-related quality of life in paediatric patients with cancer. European journal of cancer care, 28(4), e13029.

Oliveira, S. M. C., dos Santos Evangelista, V., \& da Silva, Y. M. G. P. (2017). Cuidados paliativos: prevalência de fadiga em pacientes pediátricos. Revista da Sociedade Brasileira de Clínica Médica, 15(4), 240-245.

Ouzzani, M., Hammady, H., Fedorowicz, Z., \& Elmagarmid, A. (2016). Rayyan - a web and mobile app for systematic reviews. Systematic Reviews, 5(210), 110.

Place, A. E., Stevenson, K. E., Vrooman, L. M., Harris, M. H., Hunt, S. K., O'Brien, J. E., \& Cole, P. D. (2015). Intravenous pegylated asparaginase versus intramuscular native Escherichia coli L-asparaginase in newly diagnosed childhood acute lymphoblastic leukaemia (DFCI 05-001): a randomised, open-label phase 3 trial. The lancet oncology, 16(16), 1677-1690.

Ritt-Olson, A., Miller, K., Baezconde-Garbanati, L., Freyer, D., Ramirez, C., Hamilton, A., \& Milam, J. (2018). Depressive symptoms and quality of life among adolescent and young adult cancer survivors: Impact of gender and Latino culture. Journal of adolescent and young adult oncology, 7(3), 384-388.

Rodgers, C., Wills-Bagnato, P., Sloane, R., \& Hockenberry, M. (2015). Health-related quality of life among children and adolescents during hematopoietic stem cell transplant recovery. Journal of Pediatric Oncology Nursing, 32(5), 329-336.

Rodgers, C. C., Hooke, M. C., Taylor, O. A., Koerner, K. M., Mitby, P. A., Moore, I. M., \& Pan, W. (2019). Childhood Cancer Symptom Cluster: Leukemia and Health-Related Quality of Life. In Oncology nursing forum,46(2).

Schepers, S. A., Sint Nicolaas, S. M., Haverman, L., Wensing, M., Schouten van Meeteren, A. Y., Veening, M. A., \& Grootenhuis, M. A. (2017). Real-world implementation of electronic patient-reported outcomes in outpatient pediatric cancer care. Psycho-oncology, 26(7), 951-955.

Skaczkowski, G., White, V., Thompson, K., Bibby, H., Coory, M., Pinkerton, R., \& Harrup, R. (2018). Do Australian adolescents' and young adults' experiences of cancer care influence their quality of life?. Psycho-oncology, 27(3), 990-997.

Stinson, J. N., Jibb, L. A., Nguyen, C., Nathan, P. C., Maloney, A. M., Dupuis, L. L., \& Portwine, C. (2015). Construct validity and reliability of a real-time multidimensional smartphone app to assess pain in children and adolescents with cancer. Pain, 156(12), 2607-2615.

Su, H. L., Wu, L. M., Chiou, S. S., Lin, P. C., \& Liao, Y. M. (2018). Assessment of the effects of walking as an exercise intervention for children and adolescents with cancer: A feasibility study. European Journal of Oncology Nursing, 37, 29-34.

Van Lonkhuizen, P. J., Klaver, K. M., Wefel, J. S., Sitskoorn, M. M., Schagen, S. B., \& Gehring, K. (2019). Interventions for cognitive problems in adults with brain cancer: a narrative review. European journal of cancer care, 28(3) e13088.

Varni, J. W. (2017). Scaling and Scoring of the Pediatric Quality of Live Inventory PedsQLTM. Mapi Reaserch Trust. https://eprovide.mapitrust.org/instruments/pediatric-quality-of-life-inventory.

Wayne, P. M., Lee, M. S., Novakowski, J., Osypiuk, K., Ligibel, J., Carlson, L. E., \& Song, R. (2018). Tai Chi and Qigong for cancer-related symptoms and quality of life: a systematic review and meta-analysis. Journal of Cancer Survivorship, 12(2), 256-267.

Xia, W., Li, H. C. W., Lam, K. W. K., Chung, O. K. J., Song, P., Chiu, S. Y., \& Ho, K. Y.(2018). The impact of hematologic cancer and its treatment on physical activity level and quality of life among children in mainland China. Cancer Nurs, 1(10.1097). 